\title{
Clocking the onset of bilayer coherence in a high- $T_{c}$ cuprate
}

\author{
Edoardo Baldini, ${ }^{1,2}$ Andreas Mann, ${ }^{1}$ Benjamin P. P. Mallett, ${ }^{3}$ Christopher Arrell, ${ }^{2}$ Frank van Mourik, ${ }^{2}$ Thomas Wolf, ${ }^{4}$ \\ Dragan Mihailovic, ${ }^{5}$ Jeffrey L. Tallon, ${ }^{6}$ Christian Bernhard, ${ }^{3}$ José Lorenzana, ${ }^{7}$ and Fabrizio Carbone ${ }^{1}$ \\ ${ }^{1}$ Laboratory for Ultrafast Microscopy and Electron Scattering, IPHYS, EPFL, CH-1015 Lausanne, Switzerland \\ ${ }^{2}$ Laboratory of Ultrafast Spectroscopy, ISIC, EPFL, CH-1015 Lausanne, Switzerland \\ ${ }^{3}$ Department of Physics, University of Fribourg, Chemin du Musée 3, CH-1700 Fribourg, Switzerland \\ ${ }^{4}$ Institute of Solid State Physics, Karlsruhe Institute of Technology, Postfach 3640, Karlsruhe 76021, Germany \\ ${ }^{5}$ Jozef Stefan Institute and International Postgraduate School, Jamova 39, SI-1000 Ljubljana, Slovenia \\ ${ }^{6}$ Robinson Research Institute, Victoria University of Wellington, P.O. Box 33436 Lower Hutt, New Zealand \\ ${ }^{7}$ Institute for Complex Systems - CNR, and Physics Department, University of Rome "La Sapienza", I-00185 Rome, Italy
}

(Received 6 September 2016; published 3 January 2017)

\begin{abstract}
In cuprates, a precursor state of superconductivity is speculated to exist above the critical temperature $\mathrm{T}_{\mathrm{C}}$. Here we show via a combination of far-infrared ellipsometry and ultrafast broadband optical spectroscopy that signatures of such a state can be obtained via three independent observables in an underdoped sample of $\mathrm{NdBa}_{2} \mathrm{Cu}_{3} \mathrm{O}_{6+\delta}$. The pseudogap correlations were disentangled from the response of laser-broken pairs by clocking their characteristic time scales. The onset of a superconducting precursor state was found at a temperature $\mathrm{T}_{\mathrm{ONS}}>\mathrm{T}_{\mathrm{C}}$, consistent with the temperature scale identified via static optical spectroscopy. Furthermore, the temperature evolution of the coherent vibration of the Ba ion, strongly renormalized by the onset of superconductivity, revealed a pronounced anomaly at the same temperature $\mathrm{T}_{\mathrm{ONS}}$. The microscopic nature of such a precursor state is discussed in terms of preformed pairs and enhanced bilayer coherence.
\end{abstract}

DOI: 10.1103/PhysRevB.95.024501

\section{INTRODUCTION}

The short coherence length $\left(\xi_{0} \sim 1 \mathrm{~nm}\right)$ of Cooper pairs in cuprate high-critical temperature $\left(\mathrm{T}_{\mathrm{C}}\right)$ superconductors allows for a variety of fascinating phenomena in contrast to low- $\mathrm{T}_{\mathrm{C}}$ materials, which have homogeneous superconducting (SC) properties on length scales $\xi_{0}$ of the order of several hundreds or thousands of nanometers [1]. On one hand, superconductivity and other electronic states can coexist in cuprates, with disorder tipping the balance on a local scale. On the other hand, thermal and quantum fluctuations of the SC order parameter can play an important role because of the reduced dimensionality (layered structure) of the material. Thus, understanding the interplay between SC fluctuations, inhomogeneities, competing orders, and reduced dimensionality remains a major challenge in cuprate physics.

Several temperature scales have been identified in the cuprates phase diagram [Fig. 1(a)], which presumably result from the above interplay, but whose precise meaning is far from being understood. Below $\mathrm{T}^{*}$, the pseudogap (PG) state appears [Fig. 1(a), gray circles] [2-5]. Early ideas [6,7] suggested that the PG reflected the presence of pairing correlations without long-range phase coherence. Another line of thought postulates the existence of a different kind of incipient electronic order [8-15] competing with superconductivity. Traces of such orders have been seen in different regions of the phase diagram, such as stripes [16,17], nematic order [18], time-reversal symmetry breaking [19], and incommensurate charge-density waves (CDWs) [20-22].

More recent experiments give support to the competing scenario, by showing a temperature scale for precursor effects with a doping dependence quite different from $\mathrm{T}^{*}$. For example, scanning tunneling microscopy (STM) reveals that local pairing correlations can be detected up to several tens of kelvin above $\mathrm{T}_{\mathrm{C}}$ at optimal doping [Fig. 1(a), brown squares] [23,24]. Angle-resolved photoemission spectroscopy provides a similar temperature scale $[25,26]$. In addition, local probes show the inhomogeneous nature of the phenomena [23,24,27]. Nernst effect [28] [Fig. 1(a), violet triangles] and magnetization [29] [Fig. 1(a), violet diamonds] measurements show another crossover line where precursor diamagnetic effects appear, requiring some degree of intralayer coherence.

In the case of bilayer materials, the far-infrared (FIR) c-axis conductivity provides additional information. The response can be well described by a multilayer model of coupled bilayers separated by poorly conducting regions [30-32]. In this case, precursor effects appear as an increase in the Drude spectral weight (SW) due to the coherent transport between neighboring layers. Such a bilayer coherence requires substantial intralayer coherence to set in first, and indeed it appears closer to $\mathrm{T}_{\mathrm{C}}$ [Fig. 1(a), red circles]. For example, in optimally doped (OP) materials, the onset of the bilayer coherence coincides with $\mathrm{T}_{\mathrm{C}}$ and only in the underdoped (UD) samples, the bilayer onset temperature $\mathrm{T}_{\mathrm{ONS}}$ separates from $\mathrm{T}_{\mathrm{C}}$, remaining always clearly below the $\mathrm{PG}$ temperature $\mathrm{T}^{*}[32,33]$.

A valuable strategy for disentangling the above interplay is to separate the different contributions directly in real time via pump-probe spectroscopy [34-38]. This technique allows one to perturb the equilibrium between different states with a pump pulse and to subsequently study their incoherent recovery time or the dynamics of coherent modes linked to the perturbed states. In this regard, special attention has been reserved for the transient optical response of cuprate superconductors in the near-infrared/visible spectral range [39-50]. In OP cuprates, pump-probe spectroscopy provides a simple picture, as shown in Fig. 1(b), where we collect a number of single-wavelength transient reflectivity $(\Delta R / R)$ data below $T_{C}$ (solid lines) and above $\mathrm{T}_{\mathrm{C}}$ (dashed lines) on a variety of OP materials under comparable experimental conditions [44,46,48,51]. In the normal state above $\mathrm{T}_{\mathrm{C}}$, a fast relaxation of several hundreds of femtoseconds appears. This is typically attributed to the 
(a)

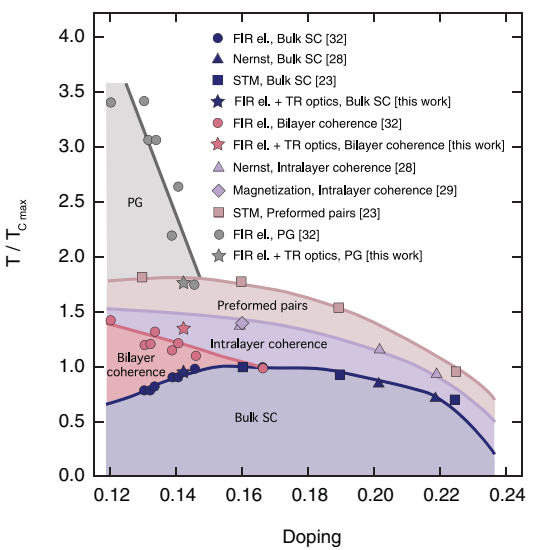

(b)

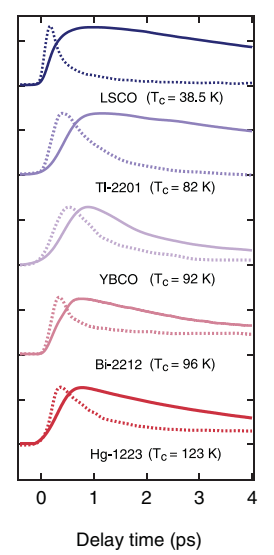

FIG. 1. (a) Phase diagram for bilayer cuprates displaying the temperature scale for SC fluctuations. The data points have been obtained by a number of experimental studies: STM [23], Nernst effect [28], magnetization [29], FIR ellipsometry [32], and this work. Symbols and colors are highlighted in the label. (b) Normalized single-wavelength transient reflectivity traces collected in the SC (solid lines) and normal (dashed lines) states on different OP cuprates under comparable experimental conditions. Data have been adapted from Refs. [44,46,48,51]. The critical temperature for each material is indicated in the figure.

cooling of a hot quasiequilibrium electron gas, giving rise to a response similar to that observed in metals [the PG signal does not manifest itself in OP cuprates; see Fig. 1(a)]. Below $\mathrm{T}_{\mathrm{C}}$, the dynamics is instead dominated by a slow relaxation component $\tau_{Q P}$ of several picoseconds, attributed to the recombination of quasiparticles (QPs) into pairs. Indeed, the relaxation dynamics of this QP response is directly related to the recovery of the SC gap detected by nonequilibrium low-energy probes [34,35,52-57].

In UD samples a more complex behavior occurs, due to the emergence of a multicomponent response. Indeed, the QP recombination dynamics across the SC gap is accompanied by an additional fast decay time $\tau_{P G}$ lasting several hundreds of femtoseconds. Since this component vanishes at $\mathrm{T}^{*}$, it has been ascribed to the recombination of the carriers subjected to PG correlations. Finally, superimposed to these relaxations, a very long decay time $\tau_{\mathrm{H}}$ of several nanoseconds appears, usually interpreted either as a pump-induced heating effect $[39,43,44,47,50,51]$ or as the signature of a photoinduced absorption from localized carriers [41,42,46]. Pioneering experiments also suggested the possibility to detect precursor effects within the PG phase of UD cuprates, by monitoring the evolution of the QP signal above $\mathrm{T}_{\mathrm{C}}$ [43]. In contrast to OP cuprates, in UD samples this component persists well above $\mathrm{T}_{\mathrm{C}}$.

Another strength of pump-probe optical spectroscopy consists in the possibility to reveal the ultrafast dynamics and the intrinsic properties of specific Raman-active bosonic collective modes [40,48,49,58-60], which are coherently excited via the impulsive stimulated Raman scattering (ISRS) process or by a long-lived perturbation of the electronic ground state [61]. Interestingly, several of these modes have strong intensity and energy anomalies at $\mathrm{T}_{\mathrm{C}}$, which suggest that

they can be used as probes of pairing correlations [40,49,59]. Similar anomalies are seen in spontaneous Raman scattering [62].

A more powerful method that goes beyond the previous description is represented by ultrafast broadband optical spectroscopy. The underlying idea behind this approach is to monitor the changes in the material optical properties with a delayed continuum probe pulse, covering a broad spectral region. This technique possesses a number of advantages over its single-wavelength counterpart. First, it allows monitoring the renormalization of the high-energy spectrum of the material under study, which depends on the dynamics of all the low-energy particle-hole excitations produced by the pump beam. In this regard, it enables one to disentangle the spectral fingerprints of the different components, adding a second dimension to time-domain spectroscopy. Moreover, the combination of the ISRS framework and the use of a broad window of detection gives access to the Raman matrix elements of the coherent bosonic modes [58-60,63,64]. When corroborated by theoretical calculations, this approach provides a very selective and quantitative estimate of the electron-boson coupling matrix elements $[59,60]$. In cuprates, a number of studies have been performed in the visible [51,58-60,65-72], mid-infrared [34], and $\mathrm{THz}$ range [53]. Among the most prominent results, we mention the detection of the coupling between the photoexcited particles and the bosonic degrees of freedom, the identification of the central role played by high-energy excitations on the Mott scale in the physics of cuprates $[51,58,68,70,71]$, the spectral separation of the QP and PG signals [34,69], and the energy exchange between the photoinduced QP response and specific infraredactive phonon modes [53].

All these arguments make ultrafast broadband optical spectroscopy a suitable candidate to study the dynamics of precursor SC effects. Here, we perform a combination of FIR ellipsometry and femtosecond broadband optical spectroscopy as a function of temperature to identify different spectroscopic features associated with the precursor SC state in an UD cuprate. As a model system, we select a high-quality single crystal of slightly UD $\mathrm{NdBa}_{2} \mathrm{Cu}_{3} \mathrm{O}_{6+\delta}$ (NBCO), which is isostructural to $\mathrm{YBa}_{2} \mathrm{Cu}_{3} \mathrm{O}_{6+\delta}(\mathrm{YBCO})$ and has a sufficiently large difference between $\mathrm{T}_{\mathrm{C}}(93.5)$ and $\mathrm{T}^{*}(170 \mathrm{~K})$ while being close to optimal doping [73]. Therefore, it is the ideal playground for identifying the spectral fingerprint of the QP signal above $T_{C}$ and for testing how its coherent phonon modes react to the possible existence of precursor phenomena.

In the nonequilibrium experiment, we reveal the $\mathrm{QP}$ response over a large spectral range, showing that this signature persists well above $T_{C}$ and vanishes only above a characteristic temperature $\mathrm{T}_{\mathrm{ONS}} \sim 130 \mathrm{~K}$. Simultaneously, the anomaly of the coherent $\mathrm{Ba}$ mode is observed at $\mathrm{T}_{\mathrm{ONS}}$ and not close to $\mathrm{T}_{\mathrm{C}}$ as in OP compounds. These findings are supported by the steady-state optical data in the FIR, which provide an independent estimate of $\mathrm{T}_{\mathrm{ONS}}$ by means of a local electric field analysis and via the temperature dependence of the $\mathrm{B}_{1 \mathrm{u}}$ $\mathrm{O}$ bending mode $[31,32]$. Our data suggest that a precursor SC state is present at these temperatures, where bilayer coherence is established among planes containing preformed pairs. 
(a)

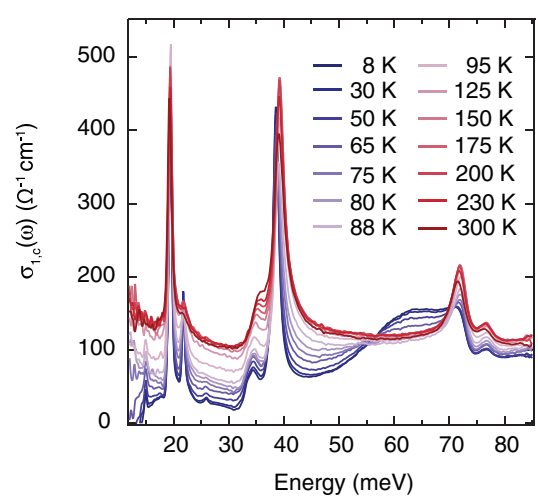

(b)

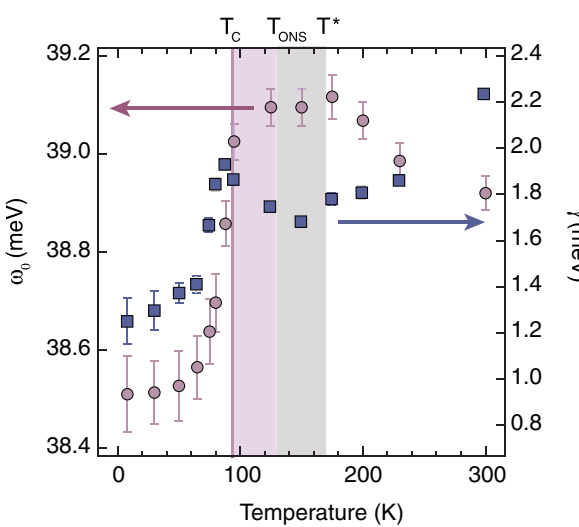

(c)

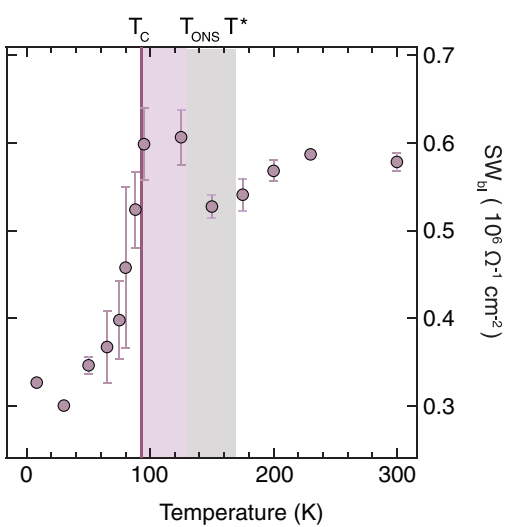

FIG. 2. (a) Real part of the c-axis optical conductivity in the FIR measured by ellipsometry as a function of temperature. (b) Temperature evolution of the $\mathrm{B}_{1 \mathrm{u}} \mathrm{O}$ bending mode frequency (violet circles) and linewidth (blue squares), showing an anomalous behavior between $\mathrm{T}_{\mathrm{C}}$ and $\mathrm{T}_{\mathrm{ONS}}$. The violet vertical line marks the value of $\mathrm{T}_{\mathrm{C}}$ and the violet (gray) shaded area highlights the temperature region characterized by incoherent pairing (PG) correlations. (c) Temperature dependence of the low-energy SW of the bilayer conductivity.

\section{RESULTS}

\section{A. Far-infrared ellipsometry}

To locate our sample in the phase diagram of Fig. 1(a), we measure the c-axis optical conductivity by means of FIR spectroscopic ellipsometry. This quantity is very sensitive to the opening of a gap in the density of states, especially near the antinodal region of the Fermi surface where a PG develops below $\mathrm{T}^{*}$ in UD samples [73,74]. This is due to the strong $\mathrm{k}$ dependence of the perpendicular hopping matrix element for the transfer of charge carriers between the $\mathrm{CuO}_{2}$ bilayer units (across the $\mathrm{BaO}$ and $\mathrm{CuO}$ chain layers). The formation of the $\mathrm{SC}$ gap below $\mathrm{T}_{\mathrm{C}}$ gives rise to an additional weaker suppression of the conductivity and to a pronounced mode at finite frequency (transverse Josephson plasma mode) [73]. The latter arises from the layered structure which is composed of two closely spaced $\mathrm{CuO}_{2}$ layers (bilayer unit) and a spacer layer that consists of two $\mathrm{BaO}$ layers and the $\mathrm{CuO}$ chain layer. This transverse plasma mode is very sensitive to the coherence of the electron transport between the $\mathrm{CuO}_{2}$ layers of the individual bilayer units and thus to the onset of SC correlations, even if they are short ranged and strongly fluctuating.

The data obtained on our NBCO single crystal are reported in Fig. 2(a) as a function of temperature. As a first step, we identify the PG and SC gap temperature scales of our sample via the analysis of the low-energy SW. The details are reported in the Supplemental Material (SM) [76]. This analysis yields $\mathrm{T}_{\mathrm{C}}=94$ and $\mathrm{T}^{*}=170 \mathrm{~K}$

Subsequently, we estimate the $\mathrm{T}_{\text {ONS }}$ temperature scale by applying the same analysis performed in Ref. [32]. In particular, we focus on the central frequency and linewidth in the optical response of the $B_{1 u}$ phonon at $\sim 39 \mathrm{meV}$. This corresponds to the bending mode of the $\mathrm{O}$ ions in the $\mathrm{CuO}_{2}$ plane, and it is also affected by the onset of the bilayer coherence which modifies the local fields on the $\mathrm{CuO}_{2}$ layers [31-33]. Figure 2(b) shows the temperature evolution of the phonon peak position and linewidth revealing a renormalization in the temperature range between 110 and
$140 \mathrm{~K}$ that signifies the onset of the bilayer coherence between these temperatures.

The $\mathrm{T}_{\text {ONS }}$ temperature scale is also estimated by a local electric field analysis of the data using the so-called multilayer model [30-32]. Within this model, $\mathrm{T}_{\text {ONS }}$ is associated with the temperature below which the bilayer conductivity starts to exhibit an increase [32,77]. For $\mathrm{T}_{\mathrm{C}}<\mathrm{T}<\mathrm{T}_{\text {ONS }}$, the condensation of pairs with a finite correlation lifetime enhances the coherence among the neighboring planes of a bilayer and thus gives rise to an increase in the low-energy SW. In the model, the real and imaginary parts of the conductivity are simultaneously fitted using the multilayer model in the energy range from 16 to $370 \mathrm{meV}$. The parameters describing the phonons are fitted at $300 \mathrm{~K}$ and essentially kept fixed at all lower temperatures, allowing only for a small reduction in the width and a blueshift of the peak energy due to thermal effects. Figure 2(c) shows the SW of the low-energy component of the bilayer conductivity. There is a significant increase in the low-energy SW below $150 \mathrm{~K}$, before it decreases again below $95 \mathrm{~K}$ due to the opening of a full SC gap (which is accompanied by a transfer of SW into a $\delta$ function at zero energy). The resulting estimate of $\mathrm{T}_{\text {ONS }}$, between 110 and $140 \mathrm{~K}$, is therefore consistent with the value extracted from the $\mathrm{B}_{1 u}$ phonon analysis.

The three temperature scales obtained for our NBCO single crystal are displayed by star symbols in the phase diagram of Fig. 1(a). The slight shifts of our values with respect to those reported in the phase diagram can be associated with a different scaling shown by NBCO single crystals compared to YBCO.

\section{B. Time-resolved broadband reflectivity}

The ability of femtosecond light excitation to set superconductivity out of equilibrium offers another route for disentangling the different temperature scales. In our timeresolved optical study, we tune the pump photon energy to be resonant with an interband charge excitation that promotes particle-hole pairs across $\mathrm{E}_{\mathrm{F}}$ towards high-energy states $[43,59]$. Afterwards, we monitor the optical reflectivity 


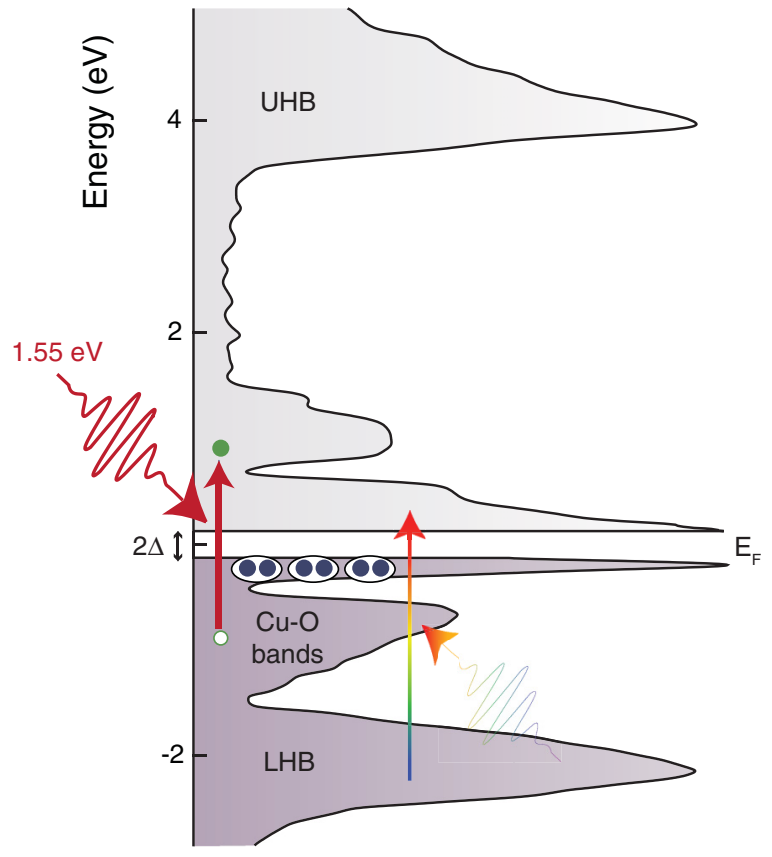

Density of states

FIG. 3. Schematic illustration of the density of states for the 123 family of cuprates. The density of states is adapted from dynamical mean field theory calculations [59]. The pump photon energy at $1.55 \mathrm{eV}$ is depicted in dark red, and the white light continuum probe is represented with a rainbow arrow. The photogenerated particle-hole pairs across $\mathrm{E}_{\mathrm{F}}$ are displayed in green, the pairs close to $\mathrm{E}_{\mathrm{F}}$ in blue. $\mathrm{UHB}=$ upper Hubbard band, LHB = lower Hubbard band.

change $\Delta R / R$ in a broad spectral region between 1.70 and $2.80 \mathrm{eV}$. This spectral range includes the absorption feature at $2.60 \mathrm{eV}$, involving occupied states in the lower Hubbard band and unoccupied states close to $E_{F}$ [59]. A pictorial illustration is offered in Fig. 3, where the one-particle density of states computed by dynamical mean field theory (adapted from ref. [59]) is shown together with the optical transitions promoted by our pump and probe pulses. Details about the steady-state optical conductivity and the description of the methods are reported in Ref. [75] and in SM [76].

Figure $4(a)$ displays $\Delta R / R$ as a function of the probe photon energy and time delay between pump and probe for some representative temperatures $(10,85,95,110,250$, and $300 \mathrm{~K})$. The temporal dynamics of $\Delta \mathrm{R} / \mathrm{R}$ at $10 \mathrm{~K}$ are displayed in Fig. 4(b) for selected probe photon energies (1.73, 2.00, and $2.50 \mathrm{eV}$ ). The signal around $2.00 \mathrm{eV}$ comprises a first, resolution-limited positive rise, followed by a second, delayed negative contribution that shows the maximum change of the response around 200 fs. Subsequently, a fast decay of the negative contribution takes place, and the response becomes positive at larger time delays. This pattern characterizes the signal across the whole spectrum, but the relative weights of the positive and negative components are strongly dependent on the probe photon energy. On top of this incoherent response an oscillation can be seen in the whole spectrum and can be ascribed to the coherent excitation of collective bosonic modes. We measure $\Delta R / R$ of our sample at 16 temperatures. The full set of data is included in the SM [76].

\section{Global fit analysis}

As a first step in our analysis, we perform a global fit of $\Delta R / R$ as a function of time in order to disentangle the contributions of the different processes occurring on our ultrafast timescale. Eleven temporal traces are selected from each map of the temperature dependence and fitted simultaneously by imposing the same time constants. At low-temperatures, a satisfactory fit of the incoherent signal can only be obtained by using three exponential functions convolved with a Gaussian response accounting for the temporal shape of the pump pulse. Whereas a resolution-limited rise characterizes the first exponential term, a delayed rise has to be used for the other two components to correctly reproduce the early dynamics. More details of the fitting procedure are provided in the SM [76]. The time constants governing the three components match the ones reported in the literature [34,39,43,44,46,50,51,53], corresponding to the PG response $\tau_{P G}$ (hundreds of femtoseconds), the QP response $\tau_{Q P}$ (several picoseconds), and the long-lived component $\tau_{H}$ (hundreds of picoseconds). The results of the fit at $10 \mathrm{~K}$ are reported in Fig. 4(b) as solid lines superimposed on the experimental traces. When iterated for all the measured $\Delta \mathrm{R} / \mathrm{R}$ maps, the global fit analysis allows one to track the separate temperature evolution of the QP and PG responses. In Fig. 4(c) we report their temperature dependences for a selected probe photon energy of $2.00 \mathrm{eV}$, at which detailed single-wavelength pump-probe studies on OP YBCO have been reported $[39,40]$. Interestingly, in our UD sample, the presence of the QP response is detected even above $\mathrm{T}_{\mathrm{C}}$, up to a temperature $\mathrm{T}_{\mathrm{ONS}} \sim 130 \mathrm{~K}$, which we associate with the onset of a precursor SC state and which is consistent with the previous equilibrium analysis. The faster PG contribution instead sets in near $\mathrm{T}^{*}$ and increases its weight down to $\mathrm{T}_{\mathrm{C}}$.

In contrast to single-wavelength pump-probe studies, our broadband probe pulse retrieves the whole $\Delta R / R$ spectrum of each contribution. The $\Delta R / R$ spectrum of the $\mathrm{QP}$ response obtained from the global fit is shown in Fig. 4(d), where, for clarity, we group the spectra below $T_{C}$ and between $T_{C}$ and $\mathrm{T}_{\mathrm{ONS}}$ in different color shadings. Figure $4(\mathrm{e})$ reports the spectrum of the $\mathrm{PG}$ component in the temperature ranges below $\mathrm{T}_{\mathrm{C}}$ and between $\mathrm{T}_{\mathrm{C}}$ and $\mathrm{T}^{*}$. By resolving the whole optical spectrum, we can observe that the QP response exhibits a sign reversal at $1.80 \mathrm{eV}$. It undergoes a continuous decrease in its amplitude with increasing temperature only in two spectral ranges, below 1.75 and around 2.00 to $2.10 \mathrm{eV}$. These results are fully consistent with previous experiments performed around $1.55[42,46,47]$ and $2.00 \mathrm{eV}[39,40]$, and demonstrate that the temperature dependence of lowenergy phenomena in cuprates can be easily tracked by these specific high-energy photons in a manner similar to low-energy probes [34,53]. Although the measured $\Delta R / R$ spectra represent a mixture of the dispersive and absorptive parts of the system's dielectric function, its identification allows one to determine the transient optical conductivity response of the system, as shown below. This capability of broadband ultrafast optical spectroscopy enables us to overcome the limitations of single-wavelength studies and provides a deeper connection with the material's electronic structure. 
(a)
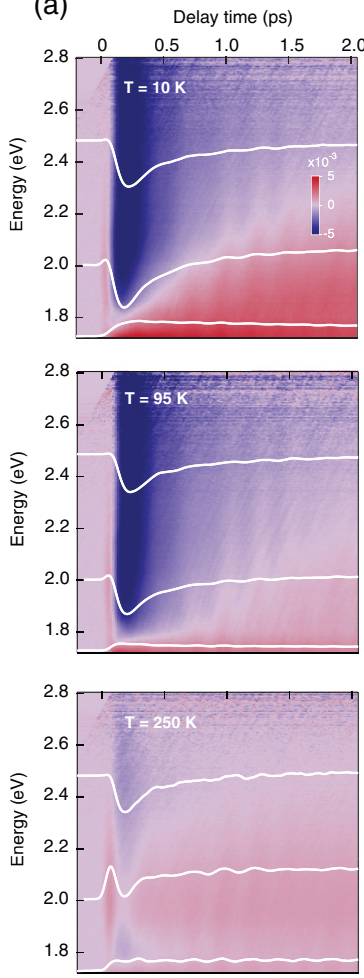

(b)
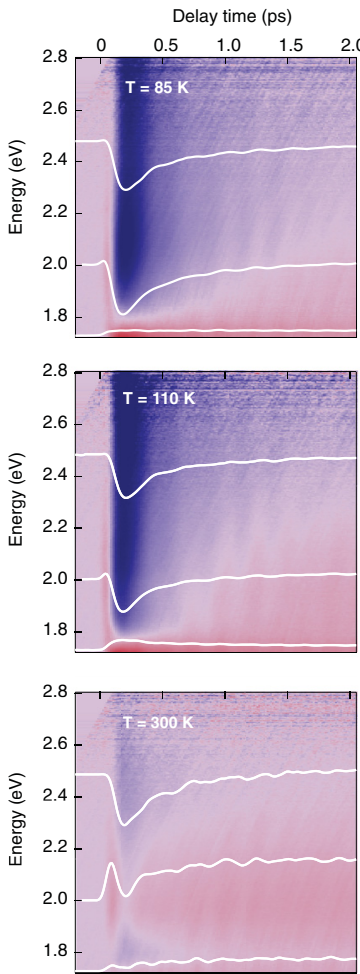

(d)
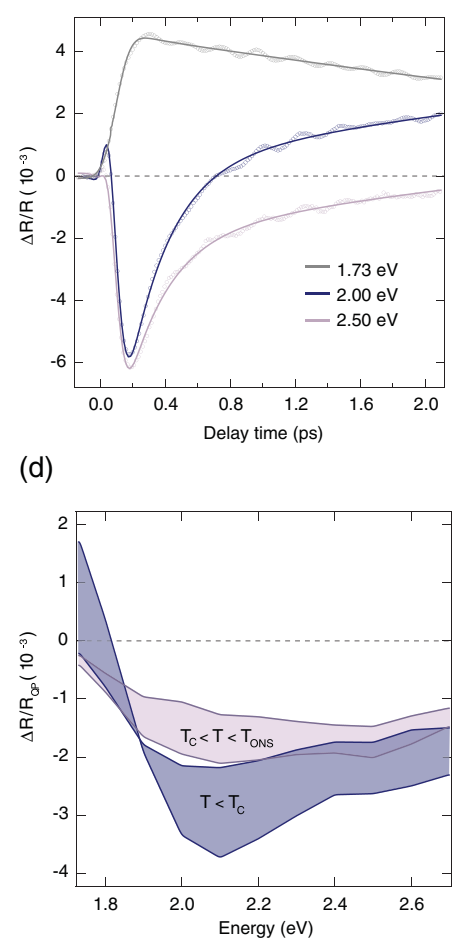

(c)

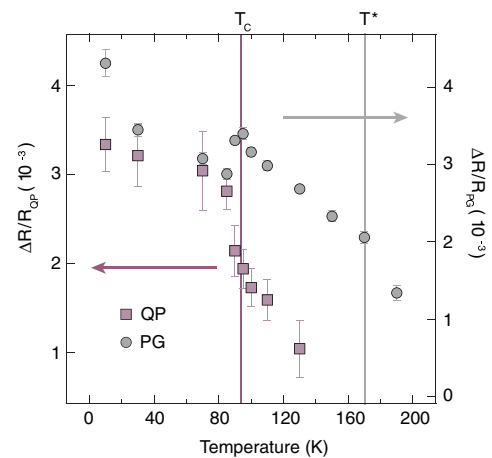

(e)

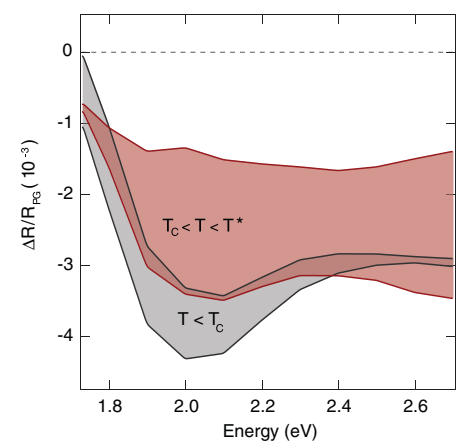

FIG. 4. (a) Transient reflectivity $\Delta \mathrm{R} / \mathrm{R}(\tau, \mathrm{E})$ as a function of the probe photon energy and time delay between pump and probe. The pump photon energy is set at $1.55 \mathrm{eV}$ and the absorbed fluence corresponds to $\sim 300 \mu \mathrm{J} / \mathrm{cm}^{2}$. The temperatures are indicated in the labels. (b) Temporal traces at $10 \mathrm{~K}$ for fixed probe photon energies of 1.73, 2.00. and $2.50 \mathrm{eV}$ are shown as dotted lines. The results of the global fit analysis are reported on top as solid lines. (c) Temperature evolution of the QP (violet squares) and PG (gray circles) contributions to $\Delta \mathrm{R} / \mathrm{R}$ at $2.00 \mathrm{eV}$ probe photon energy, as extracted from the global fit analysis. The vertical violet and gray lines identify the temperature scales $\mathrm{T}_{\mathrm{C}}$ and $\mathrm{T}^{*}$, respectively. (d),(e) Transient reflectivity spectra of the QP and PG responses, respectively, obtained from the global fit analysis. Different color shadings are used to identify the temperature regions below $\mathrm{T}_{\mathrm{C}}$ (blue) and between $\mathrm{T}_{\mathrm{C}}$ and $\mathrm{T}_{\mathrm{ONS}}$ (violet) for the $\mathrm{QP}$ response, or below $\mathrm{T}_{\mathrm{C}}$ (gray) and between $\mathrm{T}_{\mathrm{C}}$ and $\mathrm{T}^{*}$ (red) for the $\mathrm{PG}$ response.

\section{Transient optical conductivity}

As anticipated above, the use of a continuum probe can provide quantitative information on the microscopic processes affecting the visible spectral range of our cuprate. A useful quantity that can be extracted from the nonequilibrium experiment is the transient complex optical conductivity $\Delta \sigma / \sigma=$ $\Delta \sigma_{1} / \sigma_{1}+\mathrm{i} \Delta \sigma_{2} / \sigma_{2}$. This can be calculated without the need of a Kramers-Kronig transform by relying on steady-state spectroscopic ellipsometry data we measured in the visible range (reported in the SM [76]) as a starting point and performing a Drude-Lorentz analysis of the $\Delta R / R$ maps at the different temperatures. In particular, the determination of the real part $\Delta \sigma_{1} / \sigma_{1}$ gives access to the temporal evolution of the $\mathrm{SW}$ in the visible range.

In Fig. 5(a) we report $\Delta \sigma_{1} / \sigma_{1}$ at $10 \mathrm{~K}$ as a function of probe photon energy and time delay. A prominent drop is found in a wide energy range between 1.90 and $2.80 \mathrm{eV}$ at early time delays. In contrast, below $1.80 \mathrm{eV}$, a positive contribution emerges and gradually dominates the higher energy range. The response at early time delays strongly differs from the one expected from a simple transient heating of the crystal, which would show a positive sign across the whole measured spectrum (see the temperature dependence of $\sigma_{1}$ in the SM [76]). Hence, we can already assume that the pump pulse predominantly acts on the crystal as a nonthermal perturbation, creating a nonequilibrium distribution of hot carriers across $\mathrm{E}_{\mathrm{F}}$.

To unravel the effects produced by each separate ultrafast process on $\Delta \sigma_{1} / \sigma_{1}$, we fit ten temporal traces with the same model function used for $\Delta R / R$. In this way the spectral dependences of the different components can be traced, as reported in the SM [76]. The long-lived component of the response provides an increase of the SW that is mostly flat and can be reproduced from the steady-state $\sigma_{1}$ by assuming a simple heating of the crystal. In contrast, the QP and PG contributions are responsible for the pronounced decrease of the SW in the visible range (peaked around the interband transition at $2.70 \mathrm{eV}$ ) and manifest their maximum amplitude around $200 \mathrm{fs}$, suggesting the occurrence of a delayed response. This indicates that the photoinduced hot carriers decay within 200 fs to the proximity of $E_{F}$. A fraction of the energy released in this fast relaxation is conveyed to the particles subjected to SC and PG correlations, providing a channel for their excitation. Since these excited particles accumulate close to $\mathrm{E}_{\mathrm{F}}$, the final states associated with the high-energy interband transitions from the lower Hubbard band to $E_{F}$ result occupied. This explains why the broadband probe experiences a reduced absorption in the visible range due to a Pauli blocking mechanism. The SW removed in the visible range is typically transferred to low energy, contributing 
(a)

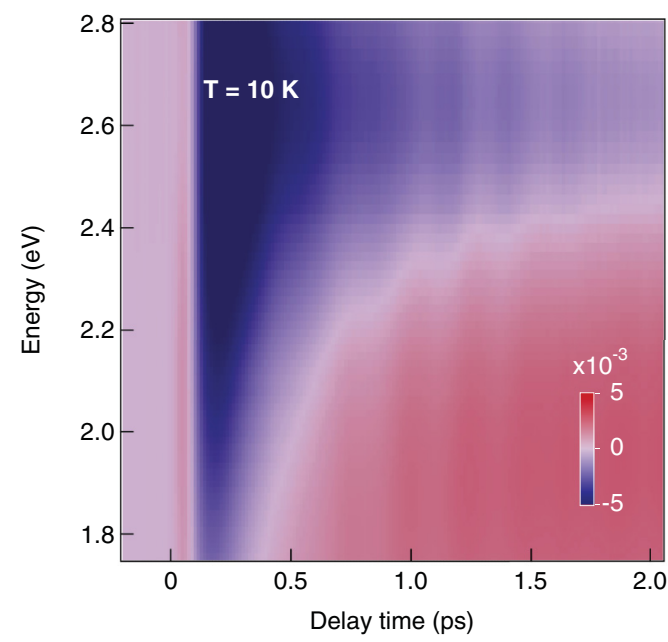

(b)

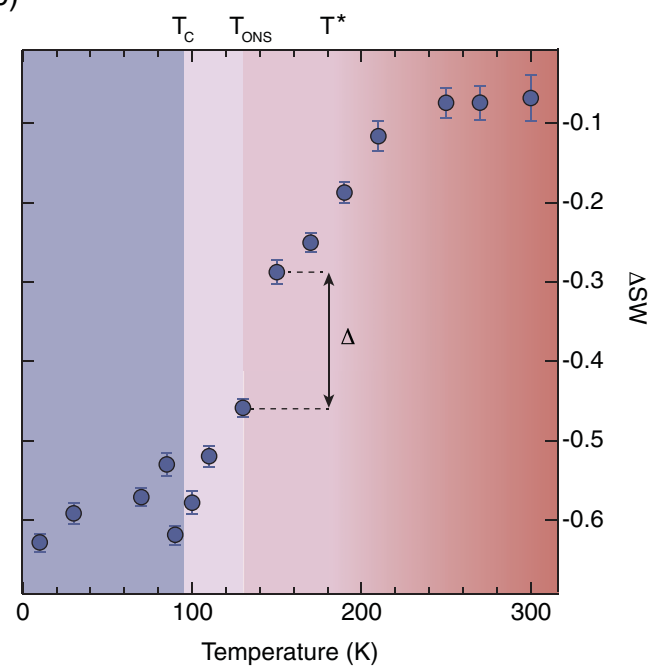

FIG. 5. (a) Calculated transient optical conductivity $\Delta \sigma_{1} / \sigma_{1}$ at $10 \mathrm{~K}$ as a function of probe photon energy and of time delay between pump and probe. (b) Temperature evolution of the nonequilibrium SW $\left(\Delta_{\mathrm{SW}}\right)$ integrated over the whole probe spectrum at 200 fs delay time. The blue shaded region highlights the temperature range where the material is superconducting, the violet region depicts the preformed state, and the gradient red region represents the crossover from the PG phase to the normal state of the material. The respective temperature scales $\mathrm{T}_{\mathrm{C}}$, $\mathrm{T}_{\mathrm{ONS}}$, and $\mathrm{T}^{*}$ are indicated on top. The $\Delta$ parameter is indicated on the graph and identifies the difference existing between the $\Delta_{\mathrm{SW}}$ at 150 and $130 \mathrm{~K}$ for a time delay of $200 \mathrm{fs}$.

significantly to the onset of a transient Drude response [53] and possibly opening new channels that were inactive under equilibrium conditions.

Finally, the determination of $\Delta \sigma_{1} / \sigma_{1}$ at all temperatures allows one to follow the temperature evolution of the change in SW $\left(\Delta_{\mathrm{SW}}\right)$ in the probed range, as displayed in Fig. $5(\mathrm{~b})$ for a time delay of $200 \mathrm{fs}$. Remarkably, $\Delta_{\mathrm{Sw}}$ displays its maximum absolute intensity in the SC state and shows a first kink in the proximity of $\mathrm{T}_{C}$, which can be related to the opening of the $\mathrm{SC}$ gap. As the temperature is increased above $\mathrm{T}_{\mathrm{C}}, \Delta_{\mathrm{SW}}$ reduces its value, which is indicative for the occurrence of a rapid crossover. A pronounced recovery of $\Delta_{\mathrm{SW}}$ is found around $\mathrm{T}_{\mathrm{ONS}}$, as the QP response from the precursor state ceases to contribute to the loss of SW in the probed spectral range. Finally, as the temperature approaches $\mathrm{T}^{*}$, a second crossover takes place and the response stabilizes around a vanishingly small constant value. Thus, by selecting the difference between $\Delta_{\mathrm{SW}}(\tau=200 \mathrm{fs})$ at 150 and $130 \mathrm{~K}$, our experiment measures the amount of SW "lost" in the visible range due to the existence of QPs in the precursor state. This quantity, which we name $\Delta$, also contains a minor contribution from the particles subjected to PG correlations, since the amplitude of their nonequilibrium response varies (although slightly) with temperature. However, already from our analysis of the incoherent response, we can conclude that signatures of the precursor state above $\mathrm{T}_{\mathrm{C}}$ emerge in the nonequilibrium experiment.

\section{E. Coherent phonon analysis}

The above analysis is complemented by the temperature dependence of the Raman-active modes which are coherently excited by the pump pulse and give rise to an oscillatory modulation across the probed spectral region. An important advantage of our technique over single-wavelength studies is that the broadband probe gives access to the energy dependent
Raman matrix elements of all collective modes affecting the visible range. When corroborated by theory, this methodology provides a very selective and quantitative estimate of the electron-phonon coupling [59,60]. Moreover, the shape of the Raman matrix elements enables us to track the temperature dependence of the coherent modes in a spectral region where they resonate with specific electronic excitations and can be clearly distinguished. To assign the collective modes present in our spectra, we perform a Fourier transform (FT) analysis of the residuals from the global fit. With this approach, we identify the presence of two separate modes influencing the high-energy electrodynamics of our UD cuprate. The Raman matrix elements of the two separate coherent modes across the probed range are shown and discussed in Fig. S14 [76]. Here, in Fig. 6(a), we report only the FT spectra around the probe photon energy of $2.10 \mathrm{eV}$ at the selected temperatures of 10 , 110,130 , and $300 \mathrm{~K}$, which clearly reveal the presence of the two separate peaks. The choice of this probe photon energy lies in the high visibility of both modes around this spectral region, as suggested by the Raman matrix elements shape.

The energies of the two peaks are $\sim 14.5$ and $\sim 19.5 \mathrm{meV}$ at $10 \mathrm{~K}$ and undergo a gradual softening as the temperature is increased. The peaks can be assigned to the totally symmetric $\left(A_{1 g}\right)$ Raman-active phonons involving the c-axis vibrations of the $\mathrm{Ba}$ and $\mathrm{Cu}$ ions, respectively. The atomic displacements are shown in Fig. 6(b). These modes have been extensively discussed in spontaneous Raman scattering and time-resolved pump-probe spectroscopy of OP YBCO [40,59,62,78]. Taking advantage of the determination of the energy dependent Raman matrix element, in our experiment we tracked the intensity of the Ba peak in the FT as a function of temperature. In Fig. 6(c) we show the results of this analysis on our slightly UD NBCO, and we compare the temperature dependence of the normalized $\mathrm{Ba}$ mode intensity with the data reported on OP YBCO [40]. The data are displayed as a function 
(a)

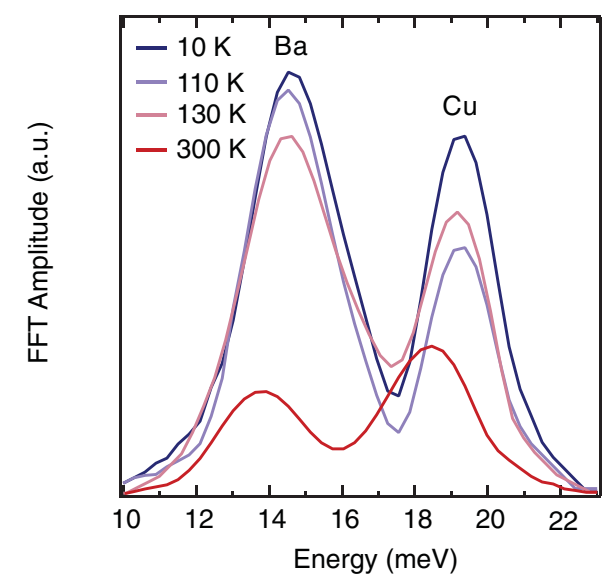

(b)

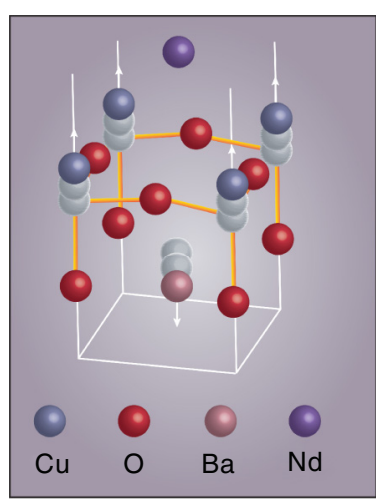

(c)

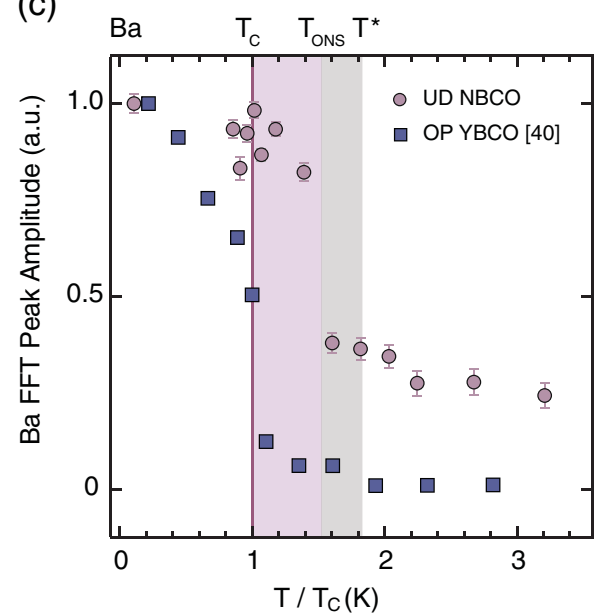

FIG. 6. (a) Fourier transform analysis of the residuals obtained from the global fit at selected temperatures. The two modes at $\sim 15$ and $\sim 19 \mathrm{meV}$ correspond to the coherent $\mathrm{A}_{1 \mathrm{~g}} \mathrm{Ba}$ and $\mathrm{Cu}$ phonons, respectively. (b) Atomic displacements of the Raman-active $\mathrm{A}_{1 \mathrm{~g}}$ phonons involving $\mathrm{c}$-axis vibrations of the $\mathrm{Ba}$ and $\mathrm{Cu}$ ions. The down-pointing arrow corresponds to the $\mathrm{Ba}$ mode, while the up-pointing arrows correspond to the $\mathrm{Cu}$ mode. (c) Temperature dependence of the $\mathrm{Ba}$ mode amplitude at $2.10 \mathrm{eV}$ of probe photon energy in OP YBCO (blue squares) and UD NBCO (violet circles). The data for OP YBCO have been extracted from Ref. [40]. The violet vertical line identifies the $\mathrm{T}_{\mathrm{C}}$ temperature scale, while the violet (gray) shaded area highlights the temperature region of NBCO which is subjected to incoherent pairing (PG) correlations.

of the normalized temperature $\mathrm{T} / \mathrm{T}_{\mathrm{C}}$, and the temperature scales $\mathrm{T}_{\text {ONS }}$ and $\mathrm{T}^{*}$ for our slightly UD NBCO sample are highlighted. Interestingly, while in OP YBCO the intensity of the $\mathrm{Ba}$ mode disappears at $\mathrm{T}_{\mathrm{C}}$, in the UD crystal it persists well above and is quenched only in the proximity of $\mathrm{T}_{\mathrm{ONS}}$ [Fig. 6(c)].

\section{DISCUSSION}

In the following, we discuss the implications of our results for the onset of the bilayer coherence.

In OP YBCO both spontaneous Raman scattering [62] and time-resolved pump-probe spectroscopy [40,59] consistently observed an anomalous intensity increase of the $\mathrm{Ba}$ mode when the crystal entered the SC phase at $\mathrm{T}_{\mathrm{C}}$ [Fig. 6(c)]. These two experimental techniques provide the same information concerning the frequency of the phonon modes, but they measure distinct states of the phonon system. Spontaneous Raman scattering measures an equilibrium response of the system. In pump-probe spectroscopy, the collective modes are instead brought into a coherent state by the pump pulse via an impulsive or displacive mechanism [61], and therefore they may be influenced by the nonequilibrium distribution of carriers and by nonlinear effects. Therefore, while in spontaneous Raman scattering the anomalous intensity increase has been explained as a superconductivity-induced resonant process (in which the Raman cross section is renormalized by self-energy effects), a different explanation has been provided by pump-probe spectroscopy. The anomalous temperature dependence of the $\mathrm{Ba}$ mode was demonstrated to follow the same behavior of the QP response, suggesting that the driving force behind the coherent excitation is represented by the change in the density of broken pairs [40]. This displacive mechanism at the origin of the coherent Ba mode has also been invoked by theoretical calculations [79]. In this scenario, the pair-breaking process instantaneously removes pairing energy from the energy balance determining the equilibrium ionic conditions, eventually triggering the coherent lattice motion. This explains why the coherent oscillation of the Ba mode in OP YBCO vanishes together with the QP signal as soon as $\mathrm{T}_{\mathrm{C}}$ is crossed. Beyond such phenomenological models, recent $a b$ initio calculations found a high sensitivity of the density of states at $\mathrm{E}_{\mathrm{F}}$ to the displacement of the Ba ion [59]. Such strong electron-phonon coupling suggests that a perturbation of the low-energy electronic states can trigger the coherent motion of the $\mathrm{Ba}$ mode. Irrespective of the details of the excitation process, there is a firm experimental evidence that the $\mathrm{Ba}$ mode is a sensitive probe of the pairing correlations.

All the above observations were reported in OP YBCO, in which there is little or no coherence among the bilayers above $\mathrm{T}_{\mathrm{C}}$ [Fig. 1(a)]. In UD samples of the 123 family, the presence of bilayer coherence above $T_{C}$ is therefore expected to play a major role in modifying the intensity anomaly of the $\mathrm{Ba}$ mode. This anomaly indicates that the Ba mode intensity is sensitive to the stabilization of the bilayer coherence, as expected from the above discussion. Hence, we attribute the large intensity change displayed by the Ba mode [Fig. 6(c)] to the establishment of the precursor SC state, consistent with the emergence of the QP component in the incoherent pumpprobe response. Such an interpretation is based on the matching between the $\mathrm{T}_{\text {ONS }}$ scale under nonequilibrium conditions and the temperature extracted from FIR ellipsometry of the same UD single crystal. Indeed, FIR ellipsometry demonstrates that the intrabilayer response becomes more coherent below $\mathrm{T}_{\mathrm{ONS}}$ and that this trend is just enhanced below $T_{C}$. In the past, the application of a magnetic field has also been shown to counteract this trend, both below $\mathrm{T}_{\mathrm{C}}$ and $\mathrm{T}_{\mathrm{ONS}}$ [32].

Although this effect is a clear evidence for enhanced SC correlations below $\mathrm{T}_{\text {ONS }}$, it does not exclude the possibility that the precursor SC state is coupled to another kind of fluctuating 
order, e.g., a CDW or spin-density wave [32]. This idea is reinforced when the portions of the YBCO phase diagram characterized by SC and CDW correlations are compared, revealing a remarkable correspondence between the $\mathrm{T}_{\mathrm{ONS}}$ temperature scales associated with the two phenomena $[32,80]$. As an example, in YBCO ortho-VIII $\left(\mathrm{T}_{\mathrm{C}}=67 \mathrm{~K}\right)$ the precursor $\mathrm{SC}$ state temperature $\mathrm{T}_{\mathrm{ONS}}=130 \mathrm{~K}$ measured by FIR ellipsometry [32] matches the temperature scale at which a CDW has been detected by resonant elastic $\mathrm{X}$-ray scattering [80]. Consistent with this scenario, recent single-wavelength pump-probe experiments proposed that the superfluid in UD cuprates can be considered as a condensate of coherently mixed particleparticle and particle-hole pairs (particle-hole quadruplets), whose origin lies in a coupled SC-CDW order parameter [81]. In the specific case of the 123 family of cuprates, one can also speculate that the coupling between the coherent $\mathrm{Ba}$ mode and the SC state is mediated by the competition among the latter and the CDW. As shown in Ref. [49], the repulsive interaction between the CDW and SC order parameters works as an efficient mechanism to establish the CDW in the SC state. Since the CDW is naturally a charged mode, it will couple strongly to all lattice phonons with the appropriate symmetry. Hence, once the CDW is established, coherent phonons will be also triggered due to their mutual coupling.

A complete understanding of this possibly intertwined precursor state setting at $\mathrm{T}_{\mathrm{ONS}}$ and of its dynamical fluctuations will become possible only upon its direct imaging with sub-picosecond time resolution. In this perspective, the devel- opment of techniques, such as time-resolved STM and cryoLorentz microscopy, is expected to play a crucial role [82-84].

\section{CONCLUSION}

In summary, using ultrafast broadband optical spectroscopy, we revealed three independent observables that suggest the emergence of a precursor SC state in the UD regime at a temperature scale $\mathrm{T}_{\mathrm{ONS}}$, such that $\mathrm{T}_{\mathrm{C}}<\mathrm{T}_{\mathrm{ONS}}<\mathrm{T}^{*}$. In particular, these observables include the persistence of the QP response up to $\mathrm{T}_{\mathrm{ONS}}$, a prominent jump in the $\mathrm{QP} \mathrm{SW}$ in the vicinity of this temperature scale, and the anomaly of the coherent $\mathrm{Ba}$ mode intensity at $\mathrm{T}_{\mathrm{ONS}}$ and not at $\mathrm{T}_{\mathrm{C}}$ as in $\mathrm{OP}$ cuprates. We interpret these phenomena in terms of precursor SC correlations, in which bilayer coherence is established among planes containing preformed pairs.

\section{ACKNOWLEDGMENTS}

Work at LUMES was supported by NCCR MUST and by the European Research Council (ERC) starting Grant No. USED258697. B.P.P.M. and C.B. acknowledge the financial support of the Swiss National Science Foundation (SNSF) through Grant No. 200020-153660 and technical support during the ellipsometry experiments at the IR beam line of the ANKA synchrotron at FZ Karlsruhe, Germany.
[1] M. Tinkham, Introduction to Superconductivity (Courier Corporation, New York, 1996).

[2] W. W. Warren, Jr., R. E. Walstedt, G. F. Brennert, R. J. Cava, R. Tycko, R. F. Bell, and G. Dabbagh, Phys. Rev. Lett. 62, 1193 (1989).

[3] H. Alloul, T. Ohno, and P. Mendels, Phys. Rev. Lett. 63, 1700 (1989).

[4] M. Takigawa, A. P. Reyes, P. C. Hammel, J. D. Thompson, R. H. Heffner, Z. Fisk, and K. C. Ott, Phys. Rev. B 43, 247 (1991).

[5] J. W. Loram, K. A. Mirza, J. R. Cooper, and W. Y. Liang, Phys. Rev. Lett. 71, 1740 (1993).

[6] V. J. Emery and S. A. Kivelson, Nature (London) 374, 434 (1995).

[7] I. Kosztin, Q. Chen, Y.-J. Kao, and K. Levin, Phys. Rev. B 61, 11662 (2000).

[8] J. Zaanen and O. Gunnarsson, Phys. Rev. B 40, 7391 (1989).

[9] V. J. Emery and S. A. Kivelson, Phys. C Supercond. 209, 597 (1993).

[10] C. Castellani, C. Di Castro, and M. Grilli, Phys. Rev. Lett. 75, 4650 (1995).

[11] L. Benfatto, S. Caprara, and C. Di Castro, Eur. Phys. J. B 17, 95 (2000).

[12] C. Nayak, Phys. Rev. B 62, 4880 (2000).

[13] A. V. Chubukov and J. Schmalian, Phys. Rev. B 57, R11085 (1998).

[14] C. M. Varma, Phys. Rev. Lett. 83, 3538 (1999).

[15] M. Capati, S. Caprara, C. Di Castro, M. Grilli, G. Seibold, and J. Lorenzana, Nat. Commun. 6, 7691 (2015).
[16] J. M. Tranquada, B. J. Sternlieb, J. D. Axe, Y. Nakamura, and S. Uchida, Nature (London) 375, 561 (1995).

[17] A. Bianconi, N. L. Saini, A. Lanzara, M. Missori, T. Rossetti, H. Oyanagi, H. Yamaguchi, K. Oka, and T. Ito, Phys. Rev. Lett. 76, 3412 (1996).

[18] V. Hinkov, D. Haug, B. Fauqu, P. Bourges, Y. Sidis, A. Ivanov, C. Bernhard, C. T. Lin, and B. Keimer, Science 319, 597 (2008).

[19] B. Fauqué, Y. Sidis, V. Hinkov, S. Pailhes, C. T. Lin, X. Chaud, and P. Bourges, Phys. Rev. Lett. 96, 197001 (2006).

[20] M. Vershinin, S. Misra, S. Ono, Y. Abe, Y. Ando, and A. Yazdani, Science 303, 1995 (2004).

[21] G. Ghiringhelli, M. L. Tacon, M. Minola, S. Blanco-Canosa, C. Mazzoli, N. B. Brookes, G. M. De Luca, A. Frano, D. G. Hawthorn, F. He, T. Loew, M. Moretti Sala, D. C. Peets, M. Salluzzo, E. Schierle, R. Sutarto, G. A. Sawatzky, E. Weschke, B. Keimer, and L. Braicovich, Science 337, 821 (2012).

[22] M. Hashimoto, E. A. Nowadnick, R.-H. He, I. M. Vishik, B Moritz, Y. He, K. Tanaka, R. G. Moore, D. Lu, and Y. Yoshida, Nat. Mater. 14, 37 (2015).

[23] K. K. Gomes, A. N. Pasupathy, A. Pushp, S. Ono, Y. Ando, and A. Yazdani, Nature (London) 447, 569 (2007).

[24] A. Yazdani, J. Phys. Condens. Matter 21, 164214 (2009).

[25] T. Kondo, Y. Hamaya, A. D. Palczewski, T. Takeuchi, J. S. Wen, Z. J. Xu, G. Gu, J. Schmalian, and A. Kaminski, Nat. Phys. 7, 21 (2011)

[26] T. Kondo, W. Malaeb, Y. Ishida, T. Sasagawa, H. Sakamoto, T. Takeuchi, T. Tohyama, and S. Shin, Nat. Commun. 6, 7699 (2015). 
[27] I. Iguchi, T. Yamaguchi, and A. Sugimoto, Nature (London) 412, 420 (2001).

[28] Y. Wang, L. Li, and N. P. Ong, Phys. Rev. B 73, 024510 (2006).

[29] L. Li, Y. Wang, S. Komiya, S. Ono, Y. Ando, G. D. Gu, and N. P. Ong, Phys. Rev. B 81, 054510 (2010).

[30] D. van der Marel and A. Tsvetkov, Czech. J. Phys. 46, 3165 (1996).

[31] D. Munzar, C. Bernhard, A. Golnik, J. Humlek, and M. Cardona, Solid State Commun. 112, 365 (1999).

[32] A. Dubroka, M. Rössle, K. W. Kim, V. K. Malik, D. Munzar, D. N. Basov, A. A. Schafgans, S. J. Moon, C. T. Lin, D. Haug, V. Hinkov, B. Keimer, T. Wolf, J. G. Storey, J. L. Tallon, and C. Bernhard, Phys. Rev. Lett. 106, 047006 (2011).

[33] E. Uykur, K. Tanaka, T. Masui, S. Miyasaka, and S. Tajima, Phys. Rev. Lett. 112, 127003 (2014).

[34] R. A. Kaindl, M. Woerner, T. Elsaesser, D. C. Smith, J. F. Ryan, G. A. Farnan, M. P. McCurry, and D. G. Walmsley, Science 287, 470 (2000).

[35] C. L. Smallwood, J. P. Hinton, C. Jozwiak, W. Zhang, J. D. Koralek, H. Eisaki, D.-H. Lee, J. Orenstein, and A. Lanzara, Science 336, 1137 (2012).

[36] W. Hu, S. Kaiser, D. Nicoletti, C. R. Hunt, I. Gierz, M. C. Hoffmann, M. Le Tacon, T. Loew, B. Keimer, and A. Cavalleri, Nat. Mater. 13, 705 (2014).

[37] R. Mankowsky, A. Subedi, M. Först, S. O. Mariager, M. Chollet, H. T. Lemke, J. S. Robinson, J. M. Glownia, M. P. Minitti, A. Frano, M. Fechner, N. A. Spaldin, T. Loew, B. Keimer, A. Georges, and A. Cavalleri, Nature (London) 516, 71 (2014).

[38] C. Giannetti, M. Capone, D. Fausti, M. Fabrizio, F. Parmigiani, and D. Mihailovic, Adv. Phys. 65, 58 (2016).

[39] S. G. Han, Z. V. Vardeny, K. S. Wong, O. G. Symko, and G. Koren, Phys. Rev. Lett. 65, 2708 (1990).

[40] W. Albrecht, T. Kruse, and H. Kurz, Phys. Rev. Lett. 69, 1451 (1992).

[41] T. N. Thomas, C. J. Stevens, A. J. S. Choudhary, J. F. Ryan, D. Mihailovic, T. Mertelj, L. Forro, G. Wagner, and J. E. Evetts, Phys. Rev. B 53, 12436 (1996).

[42] C. J. Stevens, D. Smith, C. Chen, J. F. Ryan, B. Podobnik, D. Mihailovic, G. A. Wagner, and J. E. Evetts, Phys. Rev. Lett. 78, 2212 (1997).

[43] D. Mihailovic, B. Podobnik, J. Demsar, G. Wagner, and J. Evetts, J. Phys. Chem. Sol. 59, 1937 (1998).

[44] D. C. Smith, P. Gay, D. Z. Wang, J. H. Wang, Z. F. Ren, and J. F. Ryan, Phys. C Supercond. 341-348, 2219 (2000).

[45] J. Demsar, B. Podobnik, V. V. Kabanov, T. Wolf, and D. Mihailovic, Phys. Rev. Lett. 82, 4918 (1999).

[46] D. Mihailovic, J. Demsar, R. Hudej, V. V. Kabanov, T. Wolf, and J. Karpinski, Phys. C Supercond. 341, 1731 (2000).

[47] N. Gedik, P. Blake, R. C. Spitzer, J. Orenstein, R. Liang, D. A. Bonn, and W. N. Hardy, Phys. Rev. B 70, 014504 (2004).

[48] D. H. Torchinsky, F. Mahmood, A. T. Bollinger, I. Boovi, and N. Gedik, Nat. Mater. 12, 387 (2013).

[49] J. P. Hinton, J. D. Koralek, Y. M. Lu, A. Vishwanath, J. Orenstein, D. A. Bonn, W. N. Hardy, and R. Liang, Phys. Rev. B 88, 060508 (2013).

[50] I. Madan, T. Kurosawa, Y. Toda, M. Oda, T. Mertelj, P. Kusar, and D. Mihailovic, Nat. Sci. Rep. 4, 5656 (2014).

[51] C. Giannetti, F. Cilento, S. Dal Conte, G. Coslovich, G. Ferrini, H. Molegraaf, M. Raichle, R. Liang, H. Eisaki, M. Greven,
A. Damascelli, D. van der Marel, and F. Parmigiani, Nat. Commun. 2, 353 (2011).

[52] R. P. Saichu, I. Mahns, A. Goos, S. Binder, P. May, S. G. Singer, B. Schulz, A. Rusydi, J. Unterhinninghofen, and D. Manske, Phys. Rev. Lett. 102, 177004 (2009).

[53] A. Pashkin, M. Porer, M. Beyer, K. W. Kim, A. Dubroka, C. Bernhard, X. Yao, Y. Dagan, R. Hackl, A. Erb, J. Demsar, R. Huber, and A. Leitenstorfer, Phys. Rev. Lett. 105, 067001 (2010).

[54] R. Cortés, L. Rettig, Y. Yoshida, H. Eisaki, M. Wolf, and U. Bovensiepen, Phys. Rev. Lett. 107, 097002 (2011).

[55] J. Graf, C. Jozwiak, C. L. Smallwood, H. Eisaki, R. A. Kaindl, D.-H. Lee, and A. Lanzara, Nat. Phys. 7, 805 (2011).

[56] W. Zhang, C. L. Smallwood, C. Jozwiak, T. L. Miller, Y. Yoshida, H. Eisaki, D.-H. Lee, and A. Lanzara, Phys. Rev. B 88, 245132 (2013).

[57] W. Zhang, C. Hwang, C. L. Smallwood, T. L. Miller, G. Affeldt, K. Kurashima, C. Jozwiak, H. Eisaki, T. Adachi, Y. Koike, D.-H. Lee, and A. Lanzara, Nat. Commun. 5, 4959 (2014).

[58] B. Mansart, J. Lorenzana, A. Mann, A. Odeh, M. Scarongella, M. Chergui, and F. Carbone, Proc. Natl. Acad. Sci. USA 110, 4539 (2013).

[59] D. Fausti, F. Novelli, G. Giovannetti, A. Avella, F. Cilento, L. Patthey, M. Radovic, M. Capone, and F. Parmigiani, arXiv: 1408.0888 [cond-mat.str-el].

[60] A. Mann, E. Baldini, A. Tramontana, E. Pomjakushina, K. Conder, C. Arrell, F. van Mourik, J. Lorenzana, and F. Carbone, Phys. Rev. B 92, 035147 (2015).

[61] T. E. Stevens, J. Kuhl, and R. Merlin, Phys. Rev. B 65, 144304 (2002).

[62] B. Friedl, C. Thomsen, H.-U. Habermeier, and M. Cardona, Solid State Commun. 78, 291 (1991).

[63] S. Borroni, E. Baldini, A. Mann, C. Arrell, F. van Mourik, J. Teyssier, J. Lorenzana, and F. Carbone, arXiv:1507.07193.

[64] A. Mann, E. Baldini, A. Odeh, A. Magrez, H. Berger, and F. Carbone, Phys. Rev. B 94, 115122 (2016).

[65] K. Matsuda, I. Hirabayashi, K. Kawamoto, T. Nabatame, T. Tokizaki, and A. Nakamura, Phys. Rev. B 50, 4097 (1994).

[66] H. Okamoto, T. Miyagoe, K. Kobayashi, H. Uemura, H. Nishioka, H. Matsuzaki, A. Sawa, and Y. Tokura, Phys. Rev. B 82, 060513 (2010).

[67] H. Okamoto, T. Miyagoe, K. Kobayashi, H. Uemura, H. Nishioka, H. Matsuzaki, A. Sawa, and Y. Tokura, Phys. Rev. B 83, 125102 (2011).

[68] S. Dal Conte, C. Giannetti, G. Coslovich, F. Cilento, D. Bossini, T. Abebaw, F. Banfi, G. Ferrini, H. Eisaki, M. Greven, A. Damascelli, D. van der Marel, and F. Parmigiani, Science 335, 1600 (2012).

[69] G. Coslovich, C. Giannetti, F. Cilento, S. Dal Conte, T. Abebaw, D. Bossini, G. Ferrini, H. Eisaki, M. Greven, A. Damascelli, and F. Parmigiani, Phys. Rev. Lett. 110, 107003 (2013).

[70] F. Cilento, S. Dal Conte, G. Coslovich, S. Peli, N. Nembrini, S. Mor, F. Banfi, G. Ferrini, H. Eisaki, M. K. Chan, C. J. Dorow, M. J. Veit, M. Greven, D. van der Marel, R. Comin, A. Damascelli, L. Rettig, U. Bovensiepen, M. Capone, C. Giannetti, and F. Parmigiani, Nat. Commun. 5, 4353 (2014).

[71] F. Novelli, G. De Filippis, V. Cataudella, M. Esposito, I. Vergara, F. Cilento, E. Sindici, A. Amaricci, C. Giannetti, D. Prabhakaran, S. Wall, A. Perucchi, S. Dal Conte, G. Cerullo, M. Capone, 
A. Mishchenko, M. Grüninger, N. Nagaosa, F. Parmigiani, and D. Fausti, Nat. Commun. 5, 5112 (2014).

[72] S. Dal Conte, L. Vidmar, D. Golež, M. Mierzejewski, G. Soavi, S. Peli, F. Banfi, G. Ferrini, R. Comin, B. M. Ludbrook, L. Chauviere, N. D. Zhigadlo, H. Eisaki, M. Greven, S. Lupi, A. Damascelli, D. Brida, M. Capone, J. Bonča, G. Cerullo, and C. Giannetti, Nat. Phys. 11, 421 (2015).

[73] L. Yu, D. Munzar, A. V. Boris, P. Yordanov, J. Chaloupka, T. Wolf, C. T. Lin, B. Keimer, and C. Bernhard, Phys. Rev. Lett. 100, 177004 (2008).

[74] O. K. Andersen, A. I. Liechtenstein, O. Jepsen, and F. Paulsen, J. Phys. Chem. Sol. 56, 1573 (1995).

[75] E. Baldini, A. Mann, S. Borroni. C. Arrell, F. van Mourik, and F. Carbone, Structural Dynamics 3, 064301 (2016).

[76] See Supplemental Material at http://link.aps.org/supplemental/ 10.1103/PhysRevB.95.024501 for the methods, the complete data sets of the steady-state dielectric function, the complete data sets of the pump-probe experiment as a function of temperature and fluence, the global fit analysis, the transient optical conductivity response and the coherent phonon matrix elements.

[77] J. Corson, R. Mallozzi, J. Orenstein, J. N. Eckstein, and I. Bozovic, Nature (London) 398, 221 (1999).
[78] O. V. Misochko, K. Kisoda, K. Sakai, and S. Nakashima, Phys. Rev. B 61, 4305 (2000).

[79] I. I. Mazin, A. I. Liechtenstein, O. Jepsen, O. K. Andersen, and C. O. Rodriguez, Phys. Rev. B 49, 9210 (1994).

[80] J. Chang, E. Blackburn, A. T. Holmes, N. B. Christensen, J. Larsen, J. Mesot, R. Liang, D. A. Bonn, W. N. Hardy, and A. Watenphul, Nat. Phys. 8, 871 (2012).

[81] J. P. Hinton, E. Thewalt, Z. Alpichshev, F. Mahmood, J. D. Koralek, M. K. Chan, M. J. Veit, C. J. Dorow, N. Barii, A. F. Kemper, D. A. Bonn, W. N. Hardy, R. Liang, N. Gedik, M. Greven, A. Lanzara, and J. Orenstein, Nat. Sci. Rep. 6, 23610 (2016).

[82] Y. Terada, S. Yoshida, O. Takeuchi, and H. Shigekawa, Nat. Phot. 4, 869 (2010).

[83] T. L. Cocker, V. Jelic, M. Gupta, S. J. Molesky, J. A. J. Burgess, G. De Los Reyes, L. V. Titova, Y. Y. Tsui, M. R. Freeman, and F. A. Hegmann, Nat. Phot. 7, 620 (2013).

[84] J. Rajeswari, P. Huang, G. F. Mancini, Y. Murooka, T. Latychevskaia, D. McGrouther, M. Cantoni, E. Baldini, J. S. White, A. Magrez, T. Giamarchi, H. M. Rønnow, and F. Carbone, Proc. Natl. Acad. Sci. USA 112, 14212 (2015). 